\section{Tina Welke}

\section{Tatort Deutsche Einheit}

Ostdeutsche Identitätsinszenierung im

"Tatort" des MDR

Bielefeld: transcript, 2012. - 393 S.

ISBN 978-3-8376-2018-4

(Zugl.: Wien, Univ., Diss. 2012)

Tina Welkes Studie zur ostdeutschen Identitätsinszenierung im MDR-,,Tatort“ entstand als Dissertation am Institut für Sprachwissenschaft der Universität Wien. Ausgangspunkt der Untersuchung ist die Hypothese, dass auch nach mehr als 20 Jahren „Deutscher Einheit“ deren ostdeutsche Anteile systematisch ausgeblendet bleiben oder der Osten Deutschland als Deutungsraum durch omnipräsente Schlagworte besetzt oder darauf reduziert werde (S. 12). Doch sei die „Identität im vereinigten Deutschland nach wie vor nicht nur durch den Hintergrund des Herkunftsbewusstseins geprägt, sondern auch durch die Erfahrungshorizonte aus der Zeit der Zweistaatlichkeit, und durch die Muster der gegenwärtigen Wahrnehmung von Ost und West" (S. 13). Diese wechselseitige Wahrnehmung lässt deutliche Einflüsse medialer Angebote erkennen.

In der Erinnerungskultur des Fernsehens komme dem "Tatort" eine wesentliche Rolle zu. Er sei Landeskunde, Ethnologie und Alltagsgeschichte der Republik gleichermaßen (S. 14). Für Welkes Untersuchung von 45 Tatortepisoden des MDR aus den Jahren 1992 bis 2007 werden Verlaufsprotokolle mit linguistischen Analysen verbaler Realisierungen ostdeutscher Identitätsinszenierungen sowie mit diskurs- und inhaltsanalytischen Verfahren der Filmsoziologie kombiniert (S.18). Ein Fokus liegt auf der Analyse der Figuren als Handlungs- und Funktionsträger (S.28), wobei eine Leitfrage lautet: „Wie gelingt es den Aktanten, den Transformationsprozess erfolgreich zu bewältigen?" (S. 125). Insbesondere die Methodenpluralität ermöglicht ein breites Erkenntnisspektrum in Fragen der Themenschwerpunkte, Dramaturgien, Ideologien, der nationalen, aber auch generationsspezifischen Identitätskonstruktion und der gesamtgesellschaftlichen Wirkung. Der von Tina Welke gewählte Untersuchungszeitraum umfasst eine 17 Jahre dauernde Erzählzeit, was dem Heranwachsen der ersten gesamtdeutschen Generation entspreche (S. 17)

Welke beschreibt den MDR-,Tatort“ als in 45 filmische Einzelerzählungen aufgeteilte Gesamtnarration ,über den Transformationsprozess des Osten Deutschlands“ (S. 13), die sie in
Beziehung zu bisherigen Diskursen über eine ostdeutsche Teilidentität (4. Kapitel) in Beziehung setzt. Sie unterteilt die Gesamtnarration in fünf Handlungsphasen, die bislang v. a. in der theater- und filmwissenschaftlichen Forschung, aber auch in diversen Drehbuchratgebern verwendet wurden: Exposition - Steigerung - Höhepunkt/Umschwung - Retardierung - Neutralisierung (S.13). Die einzelnen Tatortepisoden werden in der diachronen Analyse des siebten Kapitels diesen Phasen zugeordnet (S.133). Zusätzlich konstatiert Tina Welke textinterne Zäsuren durch Konfigurationswechsel des Protagonistenpaares und Durchbrechungen der räumlichen Kontinuität (S. 19). Auf der Grundlage ihrer Episodenanalyse entwickelt Tina Welke ein Phasenmodell der Thematisierung ostdeutscher Identität, das als Spiegel der jeweiligen Entwicklung des Verhältnisses zwischen Ost- und Westdeutschland fungiert. Dabei zeigen sich Veränderungen in der Thematisierung von Differenzen zwischen West und Ost, der Raumdarstellung und der Bedeutung der Auseinandersetzung mit der Vergangenheit.

Tina Welke bezeichnet die Sendeanstalt als übergeordnete Erzählinstanz (S. 12f.). Daran könnte sich eine theoretische Debatte zu Spezifika von Autorschaft im Fernsehen im Wechselspiel von Intendanz, Redaktion, Drehbuchautor und Regisseur anschließen. Sie wählt den Begriff des inkorporierten Erzählers „für alle an der Entstehung und Produktion eines Films beteiligten Personen" (S. 13).

Jedoch begrenzt schon das Reihenkonzept der regionalen Aufteilung des „Tatorts“ dieses Modell der Gesamtnarration. Wie wird denn die Ost-West-Thematik etwa in den NDRoder WDR-, ,Tatorten“ oder in anderen Krimiserien thematisiert? Lässt sich die für den MDR als charakteristisch angeführte Selbstreferentialität (S. 103) nicht auch in den Produktionen der anderen regionalen Sendeanstalten beobachten? Lässt sich die ARD als übergeordnete Erzählinstanz fassen? Welche Rolle spielen andere Programmschwerpunkte des Fernsehens, wie etwa Nostalgieshows des Unterhaltungsbereichs, für die Gesamterzählung des ostdeutschen Transformationsprozesses?

Es werden wenige Bezüge zur Forschungsliteratur etwa im Bereich der spezifischen Genrekonventionen von Krimiserien, zur Geschichte des Deutschen Fernsehkrimis und zu den Einflüssen amerikanischer Erzählweisen hergestellt. Stattdessen richtet sich das Erkenntnisinteresse u. a. auf die Präsentation gesellschaftlicher und sozialer Realität und die Gedächtnisfunktion des Fernsehens. 
Vereinzelt zeigen sich Probleme bei den verwendeten Begriffen. Inwieweit lässt sich etwa der „Tatort“ als Format bezeichnen? Hier wäre eine Einbeziehung der Formatforschung hilfreich gewesen, die auf ökonomisch orientierte Standardisierungen als Grundlage der Formatkonstruktion hinweisen. Auch die Zuordnung der Arbeit zur transmedialen Erzähltheorie ist angesichts der dortigen Reflexion der Aufteilung des Erzählens auf mehrere Medien problematisch.

Die fehlende Differenzierung zwischen Film und Fernsehen bildet ein Kernproblem der Untersuchung. Das gesamte dritte Kapitel befasst sich mit dem Film als Untersuchungsgegenstand und nicht etwa mit der Bedeutung der Fernsehforschung. Bereits in ihrer Einleitung spricht Tina Welke davon, dass der MDR mit 45 Filmen zum ARD- „Tatort“ beigetragen habe. Sie konstatiert sogar, es handele sich bei den untersuchten MDR-,Tatorten“ „um eine Form des seriellen Erzählens im Medium Film“ (S. 13). Ein Fokus der bisherigen Fernsehforschung lag jedoch darin, die Differenzen zwischen Fernseh- und Kinofilm zu beschreiben. Im dritten Kapitel und an anderen Stellen der Untersuchung steht das filmische Erzählen im Zentrum, obwohl das Programmmedium Fernsehen eine ganz andere erzählerische Vermittlung aufweist als das Kino. Auch hätte im Kapitel 5 nicht das Genre des Kriminalfilms den Ausgangspunkt der Untersuchung bilden sollen, sondern das Genre Krimiserie.

Hinsichtlich des Methodenansatzes bleibt zu fragen, ob eine detaillierte Analyse zweier Filmausschnitte tatsächlich als Nachweis fungieren kann, „dass sich die Identitätsinszenierung intendiert des Zusammenwirkens der filmischen Codes bedient" (S. 13) - wo doch jede Form der Bedeutungsvermittlung in audiovisuellen $\mathrm{Me}-$ dien auf dem Zusammenwirken von Codes basiert.

Die Analysen der MDR-„Tatort“-Episoden führen zu vielen interessanten Ergebnissen, etwa hinsichtlich intermedialer Bezüge zwischen den einzelnen Episoden, der Bindung impliziter Ideologien an unterschiedliche Figurencharakterisierungen oder der Konkretisierung gesellschaftlicher Diskurse in unterschiedlichen Dialogen. Es wäre gut gewesen, auch die Beziehungen zur restlichen "Tatort“-Entwicklung und vergleichbaren Raumkonstellationen, Themen und Figuren stärker herauszuarbeiten. Frau Welke bietet mit ihrer Untersuchung jedoch einen wichtigen Beitrag zum Thema der Vermittlung impliziter Ideologien durch das Fernsehen.

Joan Kristin Bleicher

\section{Thomas Wiedemann}

\section{Walter Hagemann}

Aufstieg und Fall eines politisch ambitionierten Journalisten und Publizistikwissenschaftlers

Köln: Halem, 2012. - 442 S.

(Theorie und Geschichte der

Kommunikationswissenschaft; 12)

ISBN 978-3-86962-074-9

(Zugl.: München, Univ., Diss., 2012)

Walter Hagemann ist mit Sicherheit die wohl schillerndste Persönlichkeit im Feld der akademischen Vertreter der Publizistikwissenschaft der 1950er Jahre. Sein Aufstieg als Journalist und Publizist in den 1920er Jahren, als Redakteur der katholischen Zeitung „Germania“ in der Weimarer Republik, seine Tätigkeit als Münsteraner Professor für Publizistikwissenschaft in den 1950er Jahren und seine Flucht in die DDR 1959 sind als Karriereweg beispiellos. Lutz Hachmeister hatte seinen Weg in seinem Buch „Theoretische Publizistik" vor Jahren bereits skizziert und diskutiert, trotzdem blieb vieles rätselhaft.

Thomas Wiedemann legt nun eine umfassende Darstellung des Lebensweges von Walter Hagemann vor, die neben einer sehr differenzierten Beschreibung seiner Biografie eine Darstellung, Reflektion und vorsichtige Wertung seiner Schriften und Positionen vornimmt. Er bedient sich dabei der Theorie der sozialen Praxis Pierre Bourdieus mit ihren zentralen Begriffen des sozialen Raumes und des kulturellen Feldes, des Habitus und des sozialen und kulturellen Kapitals. Diesen Ansatz skizziert er zu Beginn seiner Darstellung knapp und nutzt den theoretischen Ansatz mit seinen Kategorien als ein heuristisches Instrument, um sich ausführlich mit Hagemanns journalistischer Tätigkeit, seinem Einfluss auf die Politik und seinem wissenschaftlichen Werdegang zu beschäftigen.

Wiedemann gelingt eine gut lesbare, stellenweise spannende Darstellung von Hagemann. In seiner ärmlichen Kindheit und entbehrungsreichen Jugend findet er die Motivation für den Ehrgeiz und den ungeheuren Arbeitswillen Hagemanns, er beschreibt die weltanschauliche Prägung im rheinisch-katholischen Milieu, sein geschichtswissenschaftliches Studium und seine Promotion im Bereich des Historismus und seine frühe Positionierung im journalistischen und politischen Feld. Hagemann etabliert sich zunächst als Reisejournalist, was ihn durch alle Kontinente der Welt treibt und ihm dann in den 1920er Jahren Anerkennung einbringt und den Grundstock bildet für ein großes ,journalisti- 